\title{
Simultaneous expression of smooth and rough phase properties related to lipopolysaccharide in a strain of Brucella melitensis
}

\author{
R. A. BOWDEN, J. M. VERGER, MAGGY GRAYON, J. N. LIMET* $\dagger$ and G. DUBRAY
}

Laboratoire de Pathologie Infectieuse et Immunologie, Institut National de la Recherche Agronomique, 37380 Nouzilly, France and "Unite de Medecine Experimentale, Universite Catolique de Louvaine, 74 av. Hippocrate, Bruxelles, Belgium

\begin{abstract}
Summary. Brucella strains exhibit either a rough (R) or a smooth (S) colonial phase identifiable by bacteriological methods. This depends on the biosynthesis and translocation to the surface in $\mathrm{S}$ but not in $\mathrm{R}$ strains, of the O-polysaccharide chain of the lipopolysaccharide (LPS) molecule. B. melitensis biovar 1 strain EP exhibited simultaneously both $\mathrm{S}$ and $\mathrm{R}$ characteristics in relation to colonial morphology, agglutination by monospecific anti-M and anti-R sera, activity of bacteriophages lytic for rough Brucella spp. (phage $\mathrm{R} / \mathrm{C}$ ) and for smooth $B$. melitensis (phage Iz). B. melitensis strain EP expressed fewer O-chains with a similar distribution of molecular weights than $B$. melitensis reference strain $16 \mathrm{M}$ by SDSPAGE and immunoblotting, but higher amounts of R-LPS. Quantitative determination of SLPS by a turbidimetric latex inhibition immunoassay with monoclonal antibodies confirmed the limited expression of S-LPS in strain EP. As with other gram-negative bacteria, the phenomenon could be attributed to a deficiency in one step of the biosynthetic assembly of the O-chains.
\end{abstract}

\section{Introduction}

The genus Brucella includes species described as "smooth" (S) or "rough" (R) depending on the colonial morphology and related properties. Some of the methods used currently to identify Brucella isolates and to characterise and control reference strains rely upon the characteristics of the bacterial cell surface. Thus, direct observation of colonies, crystal violet staining, agglutination in the presence of acriflavine, serotyping and phage sensitivity are used to differentiate the $S$ from the $R$ phase. ${ }^{1 a}$

In Enterobacteriaceae, phase variation is related to mutation of steps in the biosynthesis of lipopolysaccharide (LPS) molecules and their translocation to the surface of the cell. ${ }^{2}$ In the $S$ colonial phase, brucella cells express the complete LPS (S-LPS) with the Ochain, plus LPS molecules without the O-chain (RLPS) ${ }^{3}$ but the R phase cells express only the R-LPS. Phase variation in brucellae ${ }^{1 \mathrm{~b}}$ is also related to the two major (A and $M$ ) antigenic specificities used in the classification of Brucella spp. ${ }^{4}$ These specificities reside in the O-chain of S-LPS. ${ }^{5}$ Although the use of the above techniques allows the correct identification of the majority of Brucella isolates as $\mathrm{S}$ or $\mathrm{R}$ phase, some

Received 12 Jan. 1993; revised version accepted 28 April 1993. $\dagger$ Present address: Facultes Universitaires Notre Dame de la Paix, 61 rue de Bruxelles, 5000 Namur, Belgium. strains do not give clear-cut results, and are, therefore, difficult to classify. Descriptions of such strains of Brucella $^{6}$ and bacteria of other genera ${ }^{7}$ have been reported but the methods used to investigate them have been limited.

The characterisation of a strain of $B$. melitensis biovar 1 of ambiguous colonial phase, strain EP, by traditional bacteriological and novel methods, together with molecular analysis of LPS by SDS-PAGE and quantitative immunoturbidimetric determination of S-LPS, showed clearly that this strain displayed an unusual level of expression of both S-LPS and R-LPS. The relative merits of the procedures available for determination of colonial phase in brucellae in the context of our observations on B. melitensis strain EP are discussed in this paper.

\section{Materials and methods}

\section{Bacterial strains}

B. melitensis strain EP, isolated from a patient with acute brucellosis, was obtained from Dr E. J. Young ${ }^{8}$ and is conserved in the Brucella culture collection, INRA-Nouzilly, France as No. BCCN 87.91. The other Brucella strains used in this study were obtained from the same collection: B. abortus S99, a smooth strain used for the elaboration of brucella antigen for 
diagnosis $;^{1 \mathrm{c}}$ B. melitensis $\mathrm{B} 115$, a rough strain used for preparation of brucellin; ${ }^{\text {dd }} B$. melitensis $16 \mathrm{M}$, the type strain of $B$. melitensis and reference of the biovar 1 of this species; ${ }^{\mathrm{e}} B$. melitensis $\operatorname{Rev}-1(\mathrm{R})$, a dissociated clone obtained from the smooth, vaccinal strain $R e v$ 1 ; and $B$. abortus 544, a smooth virulent strain, currently used for challenge in protection experiments.

\section{Bacteriological methods}

The strains were checked for purity, species and biovar characterisation by standard procedures ${ }^{1 \mathrm{a}}$ if not otherwise indicated.

Colonial morphology of strain EP was assessed in cultures grown both on Trypticase Soy Agar (bioMérieux, Marcy l'Etoile, France) supplemented with yeast extract (Difco) $0.1 \%$ (TSAYE medium) and on Blood Agar Base No. 2 (Oxoid; BAB medium). Methods for the observation of colonial morphology were those described by Alton et al. ${ }^{\mathrm{b}}$, i.e., direct observation of colonies by obliquely reflected light, acriflavine test and staining of colonies by crystal violet.

Izatnagar (Iz) phage, ${ }^{9}$ lytic for smooth $B$. melitensis strains and $\mathrm{R} / \mathrm{C}$ phage, ${ }^{10}$ lytic for rough brucellae were used to confirm the dual smooth-rough properties of strain EP. The Iz clone used in this laboratory was derived from a sample of $\mathrm{Iz}_{1}$ phage provided by M. J. Corbel in 1985. It was propagated on $\mathrm{S}$ phase $B$. melitensis strain $16 \mathrm{M}$ and has subsequently proved lytic for S phase cultures only. As most smooth strains of $B$. melitensis were found to be lysed by this phage, it was used in this study as a marker for the smooth character.

Serotyping by slide agglutination was performed with monospecific $\mathbf{A}$ and $\mathbf{M}$ polyclonal antisera prepared according to the method of Jones, ${ }^{11}$ and with an anti-R specific polyclonal serum prepared according to the method of Corbel et al. ${ }^{12}$ Agglutination patterns were interpreted as reported elsewhere. ${ }^{13}$

\section{FACS analysis}

B. melitensis strain EP was grown as indicated for PK-LPS extracts. Once harvested and inactivated, $c$. $0.5 \times 10^{9}$ cells were distributed in Eppendorf vials in which they were washed twice with phosphate buffered saline (PBS). Bacteria were then resuspended in $200 \mu \mathrm{l}$ of appropriate dilutions of monoclonal antibodies (MAbs) and incubated at $37^{\circ} \mathrm{C}$ for $1 \mathrm{~h}$. After three washings, cells were incubated with a 1 in 40 dilution of FITC-conjugated pig anti-mouse immunoglobulins (Nordic), at $37^{\circ} \mathrm{C}$ for $1 \mathrm{~h}$. Finally, the cells were washed three times, resuspended in $1 \mathrm{ml}$ of PBS and analysed. Cells incubated with the FITC conjugate alone served as controls.

The fluorescence emissions of 50000 FITC-stained brucella cells were quantified with a FACScan flow cytometer (Becton Dickinson Immunocytochemistry
Systems, Mountain View, CA, USA). The gates of the detectors were set to exclude large bacterial aggregates from the analysis.

\section{Proteinase K-LPS (PK-LPS) extracts}

To study LPS electrophoretic patterns and immunoreactivity, digested, whole-cell brucella lysates were prepared as follows. Cells from cultures grown on TSAYE or BAB slants at $37^{\circ} \mathrm{C}$ for $24 \mathrm{~h}$ were suspended in PBS containing Tween $800.05 \mathrm{w} / \mathrm{v}$ (PBS-T), $\mathrm{pH} 6.85$, and washed twice in this medium followed by resuspension at $3 \times 10^{10} \mathrm{cfu} / \mathrm{ml}$ in lysis solution (SDS $1 \%$, glycerol $2 \% \mathrm{v} / \mathrm{v}, 0.0625 \mathrm{M}$ Tris- $\mathrm{HCl}, \mathrm{pH} 6.8$ ). After boiling for $10 \mathrm{~min}$ the temperature was adjusted to $50^{\circ} \mathrm{C}$ and $0.625 \mathrm{mg}$ of proteinase $\mathrm{K}$ (Boehringer Mannheim $\mathrm{GmbH}$, Germany) was added to $1 \mathrm{ml}$ of lysate. The sample was digested at $50^{\circ} \mathrm{C}$ for $2 \mathrm{~h}$ and then at $20^{\circ} \mathrm{C}$ for $18 \mathrm{~h}$. For some assays, cell number and enzyme concentration were increased four-to-six times. PK-LPS extracts were kept frozen at $-20^{\circ} \mathrm{C}$ until required. For LPS quantitative determination, PK-LPS extracts were prepared from lyophilised cells as reported elsewhere. ${ }^{14}$

\section{$R-L P S$ and $S-L P S$ extracts}

Cells from $B$. melitensis strain B1 15 were cultured in the same medium and the conditions specified by USDA, Ames, Iowa as described previously. ${ }^{1 \mathrm{f}}$ Washed cells suspended in $0.15 \mathrm{M} \mathrm{NaCl}$ were dehydrated in acetone as for brucellin production. ${ }^{1 d}$ R-LPS was extracted from cells by the phenol-chloroform-petroleum ether-water method of Galanos et al. ${ }^{15}$

S-LPS of $B$. abortus $\mathbf{S} 99$ was prepared by the phenolwater method of Leong et al. ${ }^{\mathbf{1 6}}$

\section{$S D S-P A G E$}

PK-LPS samples were analysed by SDS-PAGE with the discontinuous buffer system of Lugtenberg ${ }^{17}$ with some modifications. ${ }^{13}$ Homogeneous separating gels $(140 \times 140 \times 0.75 \mathrm{~mm})$ of acrylamide $11 \%$ were cast and overlaid by an acrylamide $4 \%$ stacking gel. Alternatively, acrylamide 11-35\% linear gradient gels $(140 \times 140 \times 1.5 \mathrm{~mm})$ were prepared to analyse R-LPS. Ten $\mu$ l of each extract were carefully loaded and run for $120 \mathrm{~min}$ at $40 \mathrm{~mA} /$ gel for homogeneous gels, and for $300 \mathrm{~min}$ at $30 \mathrm{~mA} /$ gel for the gradient gels. The gels were stained for carbohydrates by the periodic acid-silver nitrate method of Dubray and Bézard. ${ }^{18}$

\section{Monoclonal antibodies (MAbs)}

To screen for epitopes present on the O-chain or in the core of the LPS, the A61/16C10/G12, B66/04F9 and A68/12/F12/G12 and anti-brucella S-LPS MAbs, specific for the $M, A$ and common epitopes of S-LPS, respectively, ${ }^{19}$ and the A68/03F03/D05 MAb specific for R-LPS ${ }^{20}$ were used. They were screened for 
Table. Main differential characteristics of $B$. melitensis strain EP and strain $16 \mathrm{M}$

\begin{tabular}{lcc}
\hline & \multicolumn{2}{c}{ Result with B. melitensis strain } \\
\cline { 2 - 3 } Test & $16 \mathrm{M}$ & EP \\
\hline $\begin{array}{l}\text { Observation of colonies } \\
\text { direct } \\
\text { crystal violet strain* }\end{array}$ & $\mathrm{S}$ & $\mathrm{S}$ \\
$\begin{array}{l}\text { Agglutination } \\
\text { acriflavine } \\
\text { monospecific serum } \\
\text { anti-A }\end{array}$ & - & + \\
$\quad \begin{array}{l}\text { anti-M } \\
\text { anti-R }\end{array}$ & - & + \\
$\begin{array}{l}\text { Lysis by phages } \\
\text { Iz }\end{array}$ & - & - \\
R/C & + & + \\
LPS expression level $\dagger$ & - & + \\
$\quad$ S-LPS & & + \\
R-LPS & + & + \\
\hline
\end{tabular}

$\mathrm{S}$, indicates colonies with a smooth, glossy surface.

* +, uptake; -, no uptake, of crystal violet by the colonies.

$\dagger$ For S-LPS, figures express relative amounts as determined by the immunoturbidimetric assay. For R-LPS, + and + + denote relative abundance as visualised in SDS-PAGE after silver staining.
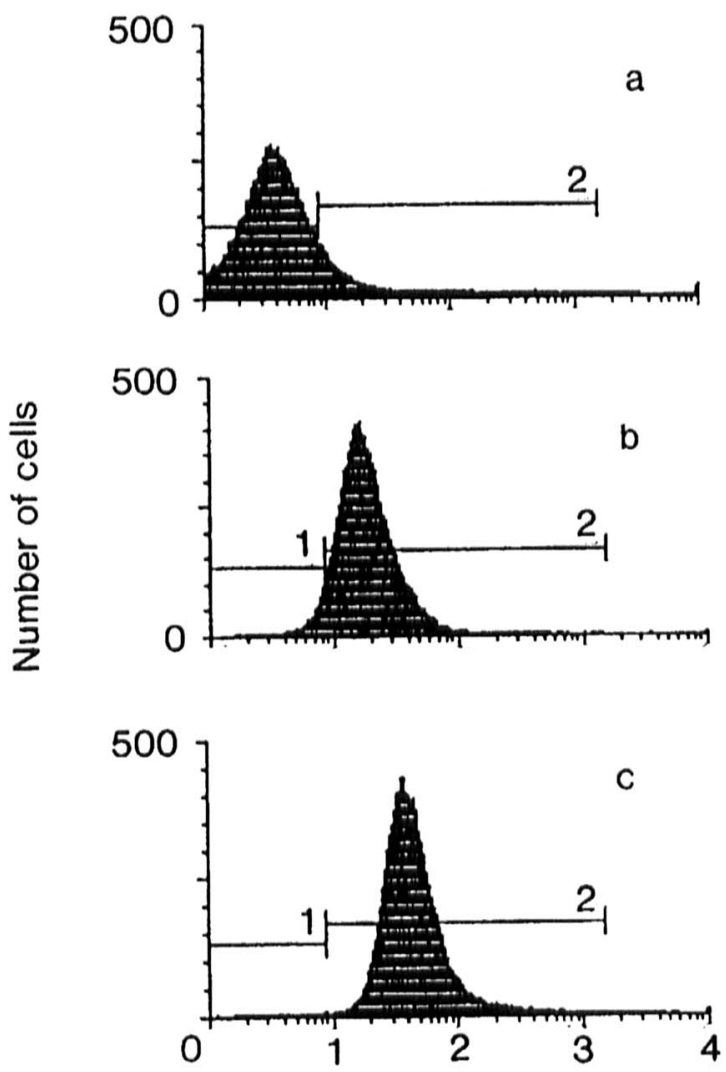

Relative fluorescence intensity $\left(\log _{10}\right)$

Fig. 1. FACS analysis of strain EP. Fluorescence histograms show the distribution of the binding of (a) control serum (FITCconjugated swine anti-mouse serum), (b) anti- R-LPS MAb A68/03F03/D05 and (c) anti-OPS MAb A68/12/F12/G12. Fluorescence intensity was plotted in relative units in a logarithmic scale.

specificity by the automated particle counting immunoassay (PACIA) and by an enzyme-linked immunosorbent assay (ELISA). ${ }^{21}$

\section{Immunoblotting}

After electrophoresis, gel slabs were soaked for $15 \mathrm{~min}$ in blotting buffer ( $39 \mathrm{~mm}$ glycine, $48 \mathrm{~mm}$ Tris, SDS $0.0375 \%$, methanol $20 \% \mathrm{v} / \mathrm{v}$ ) then transferred to $0 \cdot 2-\mu \mathrm{m}$ pore size nitrocellulose membranes (BA83 Schleicher and Schuell, Dassel, Germany) in an LKB 2117 Multiphor II electrophoresis apparatus operated for $70 \mathrm{~min}$ at $0.8 \mathrm{~mA} / \mathrm{cm}^{2}$. Blocking was by overnight incubation in skimmed milk $1 \%$ in $20 \mathrm{~mm}$ Tris, $500 \mathrm{~mm} \mathrm{NaCl}$ (TBS) at room temperature with continuous gentle agitation.

Blots were incubated for 90 min with (i) rabbit anti$M$ monospecific serum, (ii) rabbit anti-A monospecific serum, (iii) rabbit anti- $R$ monospecific serum, (iv) antiM MAb, (v) anti A-MAb or (vi) anti-R MAb at previously determined dilutions in skimmed milk $0.3 \%$-TBS.

The secondary antibody was either an anti-rabbit or anti-mouse biotinylated sheep $\mathrm{F}\left(\mathrm{ab}^{\prime}\right)$ ), (Amersham International) and incubation was at room temperature for $90 \mathrm{~min}$. Bound antibody was detected by incubating with streptavidin-peroxidase (Amersham International) at room temperature for $60 \mathrm{~min}$ and the peroxidase activity was revealed by the HRP Color Development Reagent (BioRad).

In some experiments, gels were stained for carbohydrates after blotting, as indicated elsewhere in this section.

\section{LPS-coated latex}

The details of the technique for coating were described in detail previously. ${ }^{21}$ Briefly, $50 \mu \mathrm{l}$ of K109 latex suspension (Rhône-Poulenc, Courbevoie, France) $10 \% \mathrm{w} / \mathrm{v}$ were incubated with $100 \mu \mathrm{g}$ of standard brucella S-LPS (f5) in $400 \mu 1$ of $0 \cdot 1 \mathrm{M}$ glycine, $0.17 \mathrm{M} \mathrm{NaCl}, \mathrm{pH} 9 \cdot 2$ (GBS) diluted 1 in 5, sonicated for $1 \mathrm{~min}$ at $30 \mathrm{~W}$, stabilised by adding $200 \mu \mathrm{g}$ of bovine serum albumin (BSA) in GBS 1 in 5 and incubated at room temperature for $20 \mathrm{~min}$. The pellet obtained after centrifugation at $1.2 \times 10^{4} \mathrm{~g}$ for $10 \mathrm{~min}$ was suspended in $1 \mathrm{ml}$ of SDS $1 \%$, resonicated and incubated for $1 \mathrm{~h}$ at $37^{\circ} \mathrm{C}$. It was then washed twice with $1 \mathrm{ml}$ of GBS 1 in 5, once with BSA $1 \%, 50 \mathrm{~mm}$ EDTA in GBS and finally suspended in $1 \mathrm{ml}$ of this buffer. This $0.5 \%$ stock latex suspension was stored at $-20^{\circ} \mathrm{C}$ until use. To enhance agglutination, $4 \mu \mathrm{l}$ of rheumatoid factor serum were added to $1 \mathrm{ml}$ of latex $0.05 \%$.

\section{LPS quantitative determination}

To compare the relative amounts of LPS of the EP strain and the reference $B$. melitensis strain $16 \mathrm{M}$, a turbidimetric latex inhibition immunoassay was performed. PK-LPS extracts were prepared from an equal amount (dry weight of lyophilised cells) as indicated elsewhere. ${ }^{14}$ The samples were first diluted 1 in 2 in BSA $20 \%, 0.1 \mathrm{M}$ glycine, $0.17 \mathrm{M} \mathrm{NaCl}$, Tween 80 


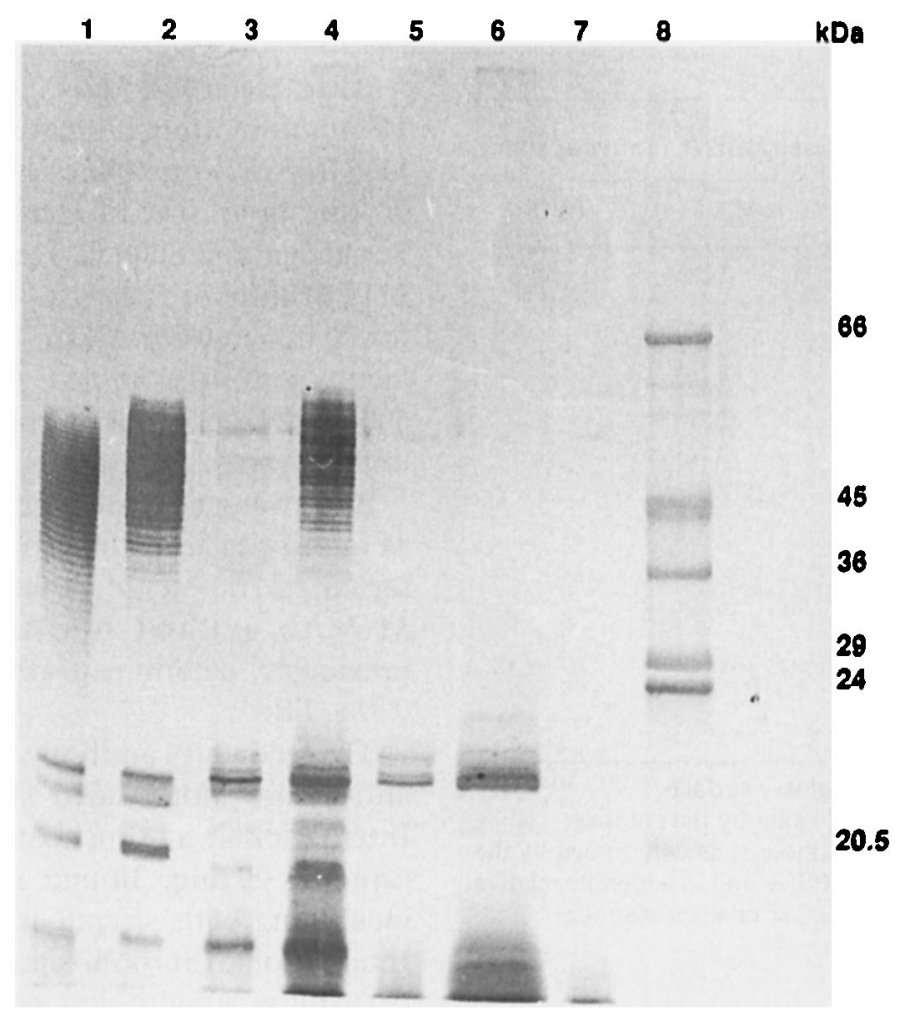

Fig. 2. SDS-PAGE of whole-cell lysates from smooth B. abortus 544 (lane 1), smooth B. melitensis 16M (2), B. melitensis EP (3 and 4) and $B$. melitensis Rev-1 rough variant (5-7). Concentrations of samples were the same except in lanes 4 and 6 in which the amount loaded was increased six-fold, and in lane 7 in which it was reduced five-fold. Lanes 5 and 6 contain purified R-LPS from B. melitensis B115. Molecular mass markers, in lane 8, were: bovine serum albumin, $66 \mathrm{kDa}$; ovoalbumin, $45 \mathrm{kDa}$; glyceraldehyde 3-phosphate dehydrogenase, $36 \mathrm{kDa}$; carbonic anhydrase, $29 \mathrm{kDa}$; trypsinogen, $24 \mathrm{kDa}$; soybean trypsin inhibitor, $20.5 \mathrm{kDa}$.

$0 \cdot 1 \%, 0.05 \mathrm{~m}$ EDTA, pH $9 \cdot 2$, (BSA $10 \%$-GBS), serially $1 \cdot 5$-fold diluted in filtered BSA $1 \%$-GBS and distributed in flat-bottomed microtitration plates (Greiner PS-Mikrotiterplate, 96K, F-form) at $40 \mu \mathrm{l} /$ well. Then, $40 \mu \mathrm{l}$ of a previously titrated dilution of $\mathrm{MAb}$ were added and the plates were shaken continuously for $30 \mathrm{~min}$ at room temperature. The latex suspension ( $40 \mu \mathrm{l} /$ well) was distributed and the plates were shaken for a few seconds. The differences (Ao-At) between the absorbances $(405 \mathrm{~nm})$ recorded immediately after latex addition (Ao) and after continuous agitation for $15 \mathrm{~min}$ (At) were calculated automatically (Titertek Multiskan MCC 340/MKII, Flow). The data were plotted against the PK-LPS dilutions, by transforming mean Ao-At of triplicates in inhibition percentages. The decrease of the absorbance of the latex suspension provoked by the agglutination of the beads, is inversely proportional to the S-LPS concentration in the PK-LPS samples.

\section{Results}

\section{Bacteriological identification}

Strain EP was identified as B. melitensis biovar 1 on the basis of the results of the tests recommended for the identification and typing of Brucella strains. Standard procedures-lysis by phages, $\mathrm{CO}_{2}$ requirement, $\mathrm{H}_{2} \mathrm{~S}$ production, dye (thionin and basic fuchsin) sensitivity and agglutination with monospecific $\mathrm{A}$ and
$M$ antisera - did not reveal any differences between strain EP and B. melitensis strain $16 \mathrm{M}$, the reference and type strain of biovar 1 of this species.

However, strain EP displayed simultaneously both smooth and rough characteristics. As any typical smooth strain of $B$. melitensis biovar 1 , it was lysed by the Iz phage and was agglutinated by the monospecific $M$ antiserum (table) but like a rough strain, it was lysed by the $\mathrm{R} / \mathrm{C}$ phage and its colonies were stained by crystal violet and were agglutinated in a 1 in 1000 solution of neutral acriflavine and by the $R$ specific serum (table).

The homogeneity of this unusual phenotype was tested on 10 randomly selected and cloned colonies, five on TSAYE medium and five on BAB medium. Whatever the medium, the 10 clones all had the typical "smooth-rough" properties described above.

To investigate these findings further, LPS of strain EP was analysed by biochemical and immunochemical methods.

\section{Examination of homogeneity by FACS analysis}

FACS analysis of strain EP showed that $99 \%$ of the population $(n=50000)$ bound to $\mathrm{MAb}$ A6812F12G12, specific for S-LPS, (mean signal = 58.5). In addition, MAb A6803F03D5, specific for RLPS, stained $95 \%$ of the cells (mean signal $=23 \cdot 15$ ). In both cases, the fluorescence histograms (fig. 1). showed a narrow, unimodal distribution. 


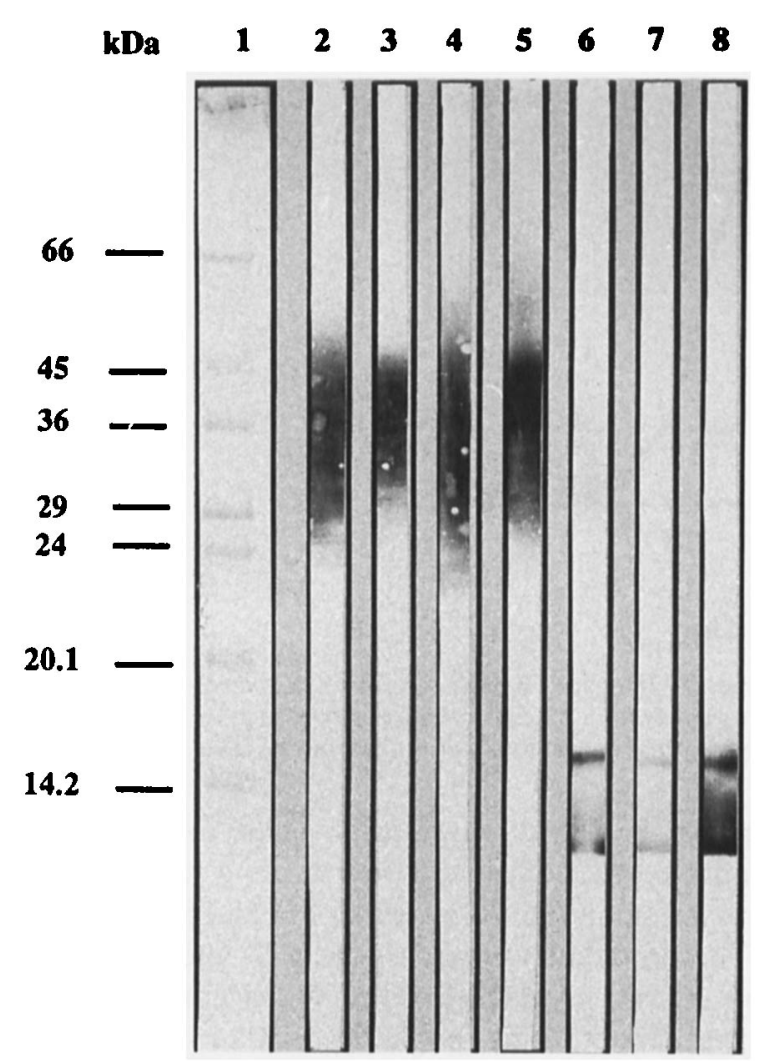

Fig. 3. Immunoblots of $B$. melitensis EP whole-cell lysate after SDSPAGE on acrylamide $11 \%$ gels. Lane 1 , molecular mass markers stained with Ponceau red after blotting; 2-7 correspond to $B$. melitensis EP whole-cell lysates; 8, purified R-LPS from B. melitensis B-115. Bands were detected by anti-M MAb (2), anti-A MAb (3), rabbit anti-M monospecific serum (4), anti-A monospecific serum (5), rabbit anti-RLPS monospecific serum (7) or by anti-R-LPS MAb (6 and 8). Material in lanes 3 and 5 were concentrated six-fold to improve the detection of the $\mathrm{A}$ antigen.

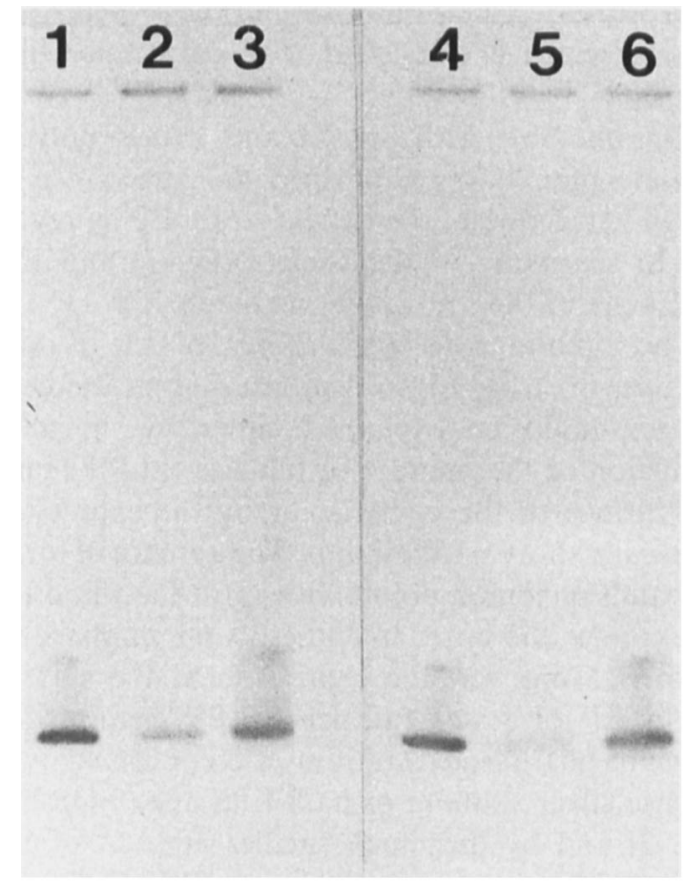

Fig. 4. Immunoblots after SDS-PAGE in acrylamide $11-35 \%$ gels of whole-cell lysates of $B$. melitensis EP (lanes 1 and 4 ); B. melitensis Rev-1 rough variant ( 2 and 5 ); purified R-LPS from rough $B$. melitensis B115 (3 and 6). Blots were detected by an anti-R-LPS $\operatorname{MAb}(\mathbf{1}, 2$ and 3$)$ or by rabbit anti-R-LPS monospecific serum $(4,5$ and 6).

\section{Molecular analysis of LPS in SDS-PAGE}

PK-LPS extracts analysed by SDS-PAGE in homogeneous acrylamide $11 \%$ gels revealed the pattern of M-predominating, $(M>A)$ brucella S-LPS, found in biovar 1 of $B$. melitensis and characterised by fine bands with intervening larger and wider bands arranged in "triplets" (fig. 2). However, the intensity of staining was noticeably lower than when PK-LPS extracts prepared from the same estimated number of cells of $B$. melitensis $16 \mathrm{M}$ were used. On the other hand, a dark-stained, diffuse band corresponding to LPS lacking O-chains (R-LPS) was present at the bottom of the gel. S-LPS bands of strain EP were found at the same position as those of strain $16 \mathrm{M}$, indicating an analogous distribution of mol. wt. In addition, some discrete bands were detected between the S-LPS and R-LPS zones.

When gradient gels of acrylamide $11-35 \%$ were used, the fast-migrating band was resolved as two wellseparated bands, and the pattern of bands of S-LPS described as above was conserved.

\section{Immunotype characterisation}

Immunoblots obtained by transfer from homogeneous $11 \%$ gels clearly revealed the pattern already described for the silver-stained bands detected in the gels. This pattern was observed with rabbit hyperimmune monospecific anti-M sera and with anti-M $\mathrm{MAb}$, thus eliminating the possibility of reaction against the core, i.e., the inner part of LPS (fig. 3).

Slide agglutination was not sufficiently sensitive to reveal the A antigens. However, the immunoblots from gels in which the amount of PK-LPS was increased six-fold confirmed the presence of A epitopes in S-LPS of strain EP (fig. 3).

Since immunoblots made from acrylamide $11-35 \%$ gradient gels increased the resolution for the fast migrating bands, these conditions were used to investigate the expression of R-LPS specificity. Anti-RLPS monospecific serum detected two well separated bands in the area of low mol. wt (fig. 4). This was similar to those produced by R-LPS from $B$. melitensis strain B115 and by a PK lysate from $B$. melitensis strain Rev-1(R). The same bands were also revealed by anti-R MAb; S-LPS bands were not detected.

No differences were observed with this technique in analysing cell preparations derived from cultures grown on either BAB or TSAYE media, although the colony surfaces looked slightly different when observed directly.

\section{Quantitative LPS determination}

A quantitative analysis was used to test the relative amount of M-type S-LPS expressed. The agglutination-inhibition method allowed immunological assay of both complete and degraded forms of S-LPS. ${ }^{14}$ When PK-LPS samples obtained from $B$. 


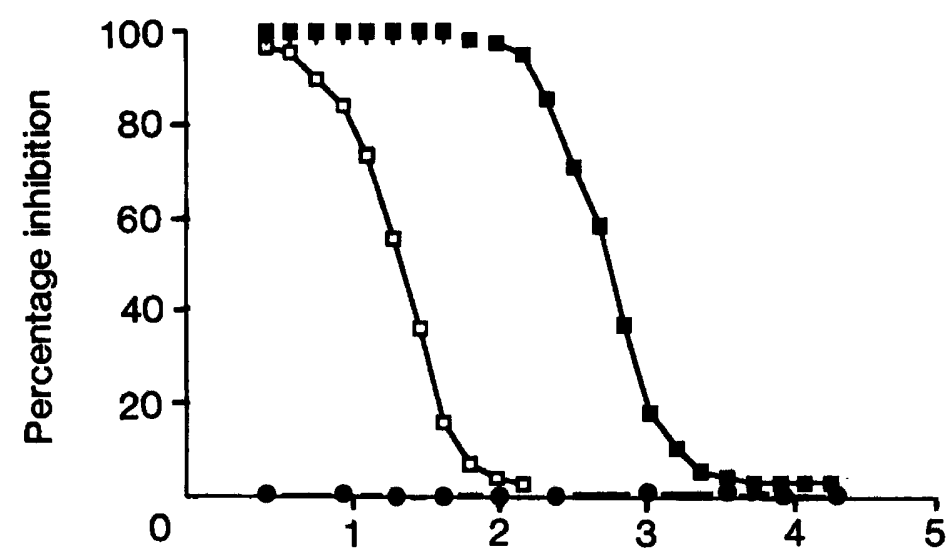

\section{Dilution $\left(\log _{10}\right)$}

Fig. 5. Agglutination-inhibition curves of B. melitensis $16 \mathrm{M},(\square)$ B. melitensis EP ( $\square$ ), and, B. melitensis Rev1 (R) whole-cell lysates ( $)$ ). Dilutions of lysates were incubated with anti-M MAb, S-LPS-coated latex was added, and the absorbances recorded and transformed into percentages of inhibition and plotted against PK-LPS dilution. Samples were at the same concentrations in terms of cellular dry-weight.

melitensis strain $16 \mathrm{M}$ were compared with similar samples from $B$. melitensis strain EP, the inhibition curves indicated that the latter contained as little as 10 times less S-LPS than the former (fig. 5). No inhibition was produced by $B$. melitensis strain Revl(R) lacking $\mathrm{O}$-chain which was included as a control. No qualitative differences were found with different culture media.

\section{Discussion}

Attention to the colonial morphology of a brucella culture is essential in the typing procedure, which is a key step in clinical microbiology. Non-smooth cultures cannot be typed with $\mathrm{A}$ and $\mathbf{M}$ monospecific sera nor with $\mathrm{S}$ phase-specific brucella phages. By direct observation, colonies of $B$. melitensis strain EP had a typical $S$ phase appearance, confirmed by agglutination with anti-M serum and by lysis with phage Iz. However, the acriflavine test and staining of colonies with crystal violet unexpectedly revealed rough characteristics which were also shown by agglutination with anti-R serum and by lysis with the brucella phage $\mathrm{R} / \mathrm{C}$. This colonial morphology in a pure culture is a rare event; except for strain EP, it was never observed in more than 3000 Brucella strains typed at this laboratory (INRA-Nouzilly).

To exclude the possibility of a mixture of populations, strain EP was checked for purity by randomly cloning 10 colonies which all had a "smooth-rough" phenotype. In addition, we studied the homogeneity of strain EP by flow cytometry. In this context, FACS analysis offered the valuable advantage of determining the distribution of epitopes in a population, but on an individual cell basis, which could give information not available from mass, colonial-level studies. We screened for expression of S- and R-related epitopes. Both S- and R-specific MAbs bound specifically to strain EP. The distribution of fluorescent events was unimodal and narrow, revealing the homogeneous expression of both parameters. Thus, FACS analysis confirmed that strain EP represents a homogeneous population and not a mixture of $R$ and $S$ cells.

The simultaneous expression of S- and R-LPS was also studied by SDS-PAGE. When compared with typical smooth and rough Brucella strains, the LPS pattern of strain EP (fig. 2) showed (i) an M-dominant structure, i.e., a succession of regularly spaced triplets as expected for a typical $\mathrm{S}$ phase strain of $B$. melitensis biovar $1,{ }^{3,9}$ and (ii) two major fast-migrating bands characteristic of R-LPS, as resolved in blots from gradient gels. The distribution of S-LPS bands in the gel was the same for both strains EP and 16M, and was consistent with those observed previously. ${ }^{3}$ The bands between the S-LPS and R-LPS zones might correspond to protease-resistant outer-membrane proteins, a conclusion which is consistent with data reported previously. ${ }^{13}$

Immunoblots with anti-M and anti-R polyclonal antisera and MAbs confirmed the mixed "smoothrough" structure of the LPS of strain EP by revealing the M-specificity of the triplets (fig. 3) and the Rspecificity of the fast-migrating bands (fig. 4).

The simultaneous accessibility of the $S$ and $R$ epitopes of strain EP to their relevant antibodies and phages could be explained either by an unusual reduction of the number of full-size S-LPS chains at the surface of the bacteria, or by the expression of atypically short S-LPS chains. The absence of low mol. wt bands on immunoblots with anti-M antibodies (fig. 3) excludes the latter hypothesis and emphasises the former. Moreover, the expression at the surface of strain EP of fewer full-size S-LPS chains than in typical S phase Brucella strains was confirmed by a less intense silver staining of its S-LPS after SDS-PAGE (fig. 2) and by the much smaller amount of S-LPS indicated by quantitative analysis of PK-SDS extracts (fig. 5).

Recently, the use of cloned DNA probes has shown that strain EP (cited as BCCN 87.91) belongs to biovar 1 of $B$. melitensis, together with the reference $B$. 
melitensis strain $16 \mathrm{M} \cdot{ }^{22}$ This confirmed the identity of strain EP as B. melitensis in spite of the atypical phage lysis results.

$S$ phase brucella cultures tend to undergo variation in colonial morphology during growth. Henry (cited by Spink $^{23}$ ) identified six different colonial types including "intermediate" and "smooth-rough" selfagglutinating ones. Huddleson (cited by Spink ${ }^{23}$ ) also described "smooth intermediate" (SI) and "mucoid" (M) colonies. Alton ${ }^{6}$ isolated B. melitensis strains from goats' milk which were found to be poor agglutinin inducers and less virulent for rabbits than typical $S$ strains. By using agglutination and absorption tests, this author deduced that the antigenic structure of the strains could be viewed as " $a R m$ ", i.e., that $R$ antigens were well exposed because $A$ and $M$ ones were present in very limited amounts. However, no explanation at a molecular level was given.

Our results, in agreement with those of Alton, ${ }^{6}$ can be related to similar findings in some Salmonella strains described by Naide et al..$^{7}$ as "semi-rough" strains, i.e., behaving morphologically as $\mathrm{S}$ phase and serologically as $\mathbf{R}$ phase or vice versa. Palva and Mäkelä ${ }^{24}$ reported a similar heterogeneity in a leaky Ra mutant of $S$. typhimurium that showed fewer S-LPS molecules of high mol. wt than current smooth isolates on SDSPAGE. The particular phenotype of Salmonella rfak mutants, ${ }^{2}$ very similar to that of strain EP, was found to be due to a reduced ability to attach O-chains because of the absence of an acetylglucosamine residue on carbon 2 of the glucose II unit of the LPS core.
As for the Salmonella "semi-rough" strains, ${ }^{7,25}$ the hypothesis of a simultaneous expression at the surface of the bacteria of relatively few full-size O-chains but numerous R-LPS "stubs" is in agreement with our SDS-PAGE, immunoblotting and quantitative results and could, therefore, account for the morphological phenotype of strain EP. The fact that full-size S-LPS molecules are expressed at a low level probably means that all the biosynthetic steps are operative but that one is limiting, either in the polymerisation of $\mathrm{O}$-chain or during the translocation of the O-chain to the core. Information is not currently available on brucella LPS biosynthesis.

Major changes in phase are relatively easy to detect in brucella cultures by the standard bacteriological methods. However, when minor variations are present in a strain, biochemical and immunochemical analysis of LPS are necessary, as demonstrated. As these changes involve both qualitative and quantitative variations of relevant epitopes, this approach can be useful in the typing of new clinical isolates, such as the EP strain, as well as in the control of reference and vaccine strains of Brucella.

R. A. Bowden was supported by SECYT-CIES and Fundacion Antorchas under a French-Argentine co-operative programme in biotechnology. Special thanks go to the academic authorities of the Facultad de Ciencias Veterinarias (UNC, Argentina) for support, and also to Dr A. Cloeckaert for providing the monoclonal antibodies. We are grateful to Dr S. Bernard for help in FACS analysis, to Dr N. Bosseray for fruitful discussions and to Dr M. Plommet for critical review of the manuscript and for encouragement. This paper is part of the Doctoral thesis of R.A.B.

\section{References}

1. Alton GG, Jones LM, Angus RD, Verger JM. Techniques for the Brucellosis laboratory. Paris, INRA. 1988: (a) 34-61; (b) 34.42 ; (c) 64 ; (d) 138 ; (e) 46 ; (f) 68 .

2. Mäkelä PH, Stocker BAD. Genetics of lipopolysaccharide. In: Rietschel ET (ed) Chemistry of endotoxin (Handbook of endotoxin, 1) Amsterdam, Elsevier. 1984: 59-137.

3. Garin-Bastuji B, Bowden RA, Dubray G, Limet JN. Sodium dodecyl sulfate-polyacrylamide gel electrophoresis and immunoblotting analysis of smooth-lipopolysaccharide heterogeneity among Brucella biovars related to $\mathrm{A}$ and $\mathbf{M}$ specificities. J Clin Microbiol 1990; 28 : 2169-2174.

4. Wilson GS. Brucella. In: Topley and Wilson's Principles of bacteriology and immunity, vol I, 5th ed. London, E. Arnold Publishing Ltd. 1964: 992-1018.

5. Cherwonogrodzky JW, Perry MB, Bundle DR. Identification of the $A$ and $M$ antigens of Brucella as the O-polysaccharides of smooth lipopolysaccharides. Can J Microbiol 1987; 33: 979-981.

6. Alton GG. The occurrence of dissociated strains of Brucella melitensis in the milk of goats in Malta. J Comp Pathol Ther 1960; 70: 10-17.

7. Naide $Y$, Nikaido $H$, Mäkelä $P H$, Wilkinson $R G$, Stocker BAD. Semirough strains of Salmonella. Proc Natl Acad Sci 1965; 53: $147-153$.

8. Young EJ, Gomez CI, Yawn DH, Musher DM. Comparison of Brucella abortus and Brucella melitensis infections of mice and their effect on acquired cellular resistance. Infect Immun 1979; 26: 680-685.

9. Corbel MJ, Tolari F, Yadava VK. Characterization of a new phage lytic for both smooth and non-smooth Brucella species. Res Vet Sci 1988; 44: 45-49.

10. Corbel MJ, Thomas EL. The Brucella-phages: their properties

characterisation and applications. Central Veterinary Laboratory Ministry of Agriculture, Fisheries and Food, Middlesex, Pinner, 1980.

11. Jones LM. A recommended method for the preparation of monospecific Brucella sera. Bull WHO 1958; 19: 177-186.

12. Corbel MJ, Gill KPW, Thomas EL. Methods for the identification of Brucella. Ministry of Agriculture, Fisheries and Food, Middlesex, Pinner, 1978.

13. Dubray G, Limet JN. Evidence of heterogeneity of lipopolysaccharides among Brucella biovars in relation to $\mathrm{A}$ and $\mathbf{M}$ specificities. Ann Inst Pasteur/Microbiol 1987; 138: 27-37.

14. Bowden RA, Van Broeck J, Dubray G, Limet JN. A turbidimetric latex inhibition immunoassay for detergent solubilized-lipopolysaccharide: application to Brucella cells. J Microbiol Methods 1992; 16: 297-306.

15. Galanos C, Lüderitz $O$, Westphal O. A new method for the extraction of R-lipopolysaccharides. Eur J Biochem 1969; 9: 245-249.

16. Leong D, Diaz R, Milner K, Rudbach J, Wilson JB. Some structural and biological properties of Brucella endotoxin. Infect Immun 1970; 1: 174-182.

17. Lugtenberg B, Meijers J, Peters R, Van der Hoek P, Van Alphen L. Electrophoretic resolution of the major outer membrane protein of Escherichia coli $\mathrm{K} 12$ into four bands. FEBS Lett 1975; 58: 254-258.

18. Dubray G, Bézard G. A highly sensitive periodic acid-silver stain for 1,2 diol groups of glycoproteins and polysaccharides in polyacrylamide gels. Anal Biochem 1982; 119: 325-329.

19. Limet JN, Bosseray N, Garin-Bastuji B, Dubray G, Plommet $M$. Humoral immunity in mice mediated by monoclonal antibodies directed against the $\mathbf{A}$ and $\mathbf{M}$ antigens of Brucella. J Med Microbiol 1989; 30: 37-43.

20. Cloeckaert A, De Wergifosse P, Dubray G, Limet JN. Identifi- 
cation of seven surface-exposed Brucella outer membrane proteins by use of monoclonal antibodies: immunogold labelling for electron microscopy and enzyme-linked immunosorbent assay. Infect Immun 1990; 58: 3980-3987.

21. Limet JN, Berbinschi A, Cloeckaert A, Cambiaso CL, Masson PL. Longitudinal study of brucellosis in mice by immunoassay of lipopolysaccharide-related antigens in blood and urine. J Med Microbiol 1988; 26: 37-45.

22. Grimont F, Verger JM, Cornelis P et al. Molecular typing of Brucella with cloned DNA probes. Res Microbiol 1992; 143: $55-65$.
23. Spink WW. The Brucella cell. In: The nature of brucellosis. Minneapolis, The University of Minnesota Press. 1956: $28-64$.

24. Palva T, Mäkelä PH. LPS heterogeneity in Salmonella typhimurium analysed by SDS polyacrylamide gel electrophoresis. Eur J Biochem 1980; 107: 137-143.

25. Lüderitz $\mathrm{O}$, Westphal $\mathrm{O}$, Staub $\mathrm{AM}$, Nikaido $\mathrm{H}$. Isolation and chemical and immunological characterization of bacterial lipopolysaccharides In: Weinbaum G, Kadis S, Ajl S (eds) Microbial toxins, vol 4, Bacterial endotoxins. New York and London, Academic Press. 1971: 145-223. 